\title{
Derivation of some contemporary scales to measure adolescent risk-taking in Canada
}

\author{
Jonathan L. Kwong ${ }^{1}$ - Don A. Klinger ${ }^{2}$ - Ian Janssen ${ }^{1,3} \cdot$ William Pickett ${ }^{1,4}$
}

Received: 23 March 2017/Revised: 7 September 2017 / Accepted: 13 October 2017/Published online: 25 October 2017

(c) The Author(s) 2017. This article is an open access publication

\begin{abstract}
Objectives To derive a contemporary series of composite indicators of adolescent risk-taking, inspired by the US CDC Framework and Problem Behaviour Theory.

Methods Factor analyses were performed on 28-risk behaviours in a nationally representative sample of 30,096 Grades 6-10 students from the 2014 Canadian Health Behaviour in School-aged Children study.

Results Three composite indicators emerged from our analysis: (1) Overt Risk-Taking (i.e., substance use, caffeinated energy drink consumption, fighting, and risky sexual behaviour), (2) Aversion to a Healthy Lifestyle (i.e., physical inactivity and low fruit and vegetable consumption), and (3) Screen Time Syndrome (i.e., abnormally high screen time use combined with unhealthy snacking). These three composite indicators of risk-taking were observed consistently with strong psychometric properties across different grade groups $(6-8,9-10)$.

Conclusions The three composite indicators of adolescent risk-taking each draw from multiple domains within the CDC framework, and support a novel, empirically directed approach of conceptualizing multiple risk behaviours among adolescents. The measures also highlight the
\end{abstract}

William Pickett

will.pickett@queensu.ca

1 Department of Public Health Sciences, Queen's University, Caruthers Hall, 2nd Floor, Kingston, ON K7L 3N6, Canada

2 Faculty of Education, Queen's University, Kingston, ON, Canada

3 School of Kinesiology and Health Studies, Queen's University, Kingston, ON, Canada

4 Department of Emergency Medicine, Queen's University, Kingston, ON, Canada breadth and diversity of risk behaviour engagement among Canadian adolescents. Research and preventive interventions should simultaneously consider the related behaviours within each of these composite indicators.

Keywords Adolescent · Risk behaviour · Problem Behaviour Theory

\section{Introduction}

Adolescent risk-taking behaviours are well-established causes of illness and injury (Turner et al. 2004). 'Problem Behaviour Theory' is foundational to modern adolescent risk research, and suggests that adolescents develop and exhibit risk-taking behaviours in related groups based on a variety of upstream determinants (called risk factors) (Jessor 1991, 2014). Such risk factors lead to the development of multiple risk behaviours that cluster together in predictable patterns within populations of young people (Pickett et al. 2002; De La Haye et al. 2014; Pfortner et al. 2015). Research and ongoing surveillance efforts in the field of adolescent risk-taking should, therefore, focus on these behaviours, both individually and in composite, to inform prevention efforts aimed specifically at adolescents. While other studies consider "risk behaviours" in the context of intent or motivation (Pérez Fuentes et al. 2016), this study uses the term to define the true action itself that poses potential harm to adolescents (e.g., Pickett et al. 2002; Riesch et al. 2013).

Over 2 decades ago, the US Centers of Disease Control and Prevention (CDC) created a risk behaviour framework that classified adolescent risk behaviours using evidence derived from the US Youth Risk Behaviour Survey (Kann et al. 2016). This long-standing framework categorizes risk 
behaviours across six domains that are associated with leading causes of morbidity and mortality among American youths: tobacco use, alcohol and illicit substance use, highrisk sexual behaviour, injury-prone and violent behaviour, unhealthy dietary patterns, and physical inactivity (Kann et al. 2016). These domains, informed by peer-reviewed literature (Brener et al. 2004), were established by consensus and are regularly updated to include emergent types of adolescent risk behaviours. Although this framework was initially developed for surveillance and policy development, it is probably the most frequently applied tool for population-based and clinical activities because of its broad consideration of varying behaviours associated with adolescent health.

There is a rich literature available that describes interrelationships between adolescents' risk behaviours and their potential effects on a variety of negative health outcomes (Yarber and Parrillo 1992; Lytle 2002; Schane et al. 2010; Spring et al. 2012; Thompson et al. 2014); however, such studies rarely consider the full complement of behaviours outlined within the CDC framework. This is particularly true in our own country of Canada. As a consequence, there may be an incomplete conceptual understanding of contemporary adolescent risk-taking behaviour and how such behaviours tend to develop and occur concurrently. For example, a large body of literature on adolescent risk-taking focuses on behaviours found in the stereotypical delinquent adolescent (i.e., the CDC domains of: alcohol and illicit substance use, tobacco use, and high-risk sexual behaviours) (Lindberg et al. 1995; Turner et al. 2004). Although these behaviours are suggestive of a high-risk lifestyle, they may also be related conceptually (and mathematically) to risk behaviours found in other domains within the CDC framework. In addition, there may be new risk behaviours within the $\mathrm{CDC}$ domains reflective of more contemporary patterns [e.g., e-cigarettes in tobacco use, and caffeinated energy drinks in alcohol and illicit substance use (Seifert et al. 2011; Goniewicz et al. 2016)], that are not included in these traditionally defined risk behaviour clusters. Behaviours from those and other domains in the CDC framework may be interrelated in different ways that reveal new patterns of risk behaviour. Finally, it is possible that adolescents in Canada engaged in risk-taking in ways that are unique from their American counterparts, and most of the existing empirical research in this field has been concentrated in the United States (Basen-Engquist et al. 1996; Riesch et al. 2013).

We had the opportunity, through analyses that involved both exploratory and confirmatory methods and a large population-based study of Canadians adolescents (Currie et al. 2012), to perform a contemporary analysis of adolescent risk-taking in Canada. Our objective was to explore inter-relationships between contemporary expressions of adolescent risk-taking, yet inspired by the long-standing CDC framework, and as a result, to create and validate new composite indicators of adolescent risk behaviours in a Canadian adolescent population. Our hope was that this would provide valuable new information in support of preventive initiatives in our country, and perhaps elsewhere.

\section{Methods}

\section{Study base and sampling}

Our study was based on Canadian records $(N=30,096)$ from the Health Behaviour in School-aged Children study (HBSC), a World Health Organization collaborative cross-national study (Currie et al. 2012). Cycle 7 of the Canadian HBSC evaluated health outcomes, attitudes, and behaviours using a confidential questionnaire administered to students from 377 schools during the 2013-14 academic year. The Canadian HBSC followed an international sampling protocol. Classes within selected schools were selected for participation using a weighted probability technique to ensure proportional representation based on the 10 Canadian provinces and three territories and the following demographic characteristics: urban-rural geographic location, language of instruction, religion, and community size. The target age range of students was 11-15 years, which typically corresponds to Grades 6-10 in Canada (Freeman et al. 2016). Grades 6-8 students were given a condensed survey that omitted questions of a more sensitive-nature (i.e., illicit drug use and sexual behaviour). Students enrolled in private, special needs, on-reserve, or faith schools (other than publicly funded Roman Catholic Schools) were not included; they represent $<7 \%$ of the Canadian student population in this age range (Van Pelt et al. 2015). Survey weights were applied to ensure that the sample was generalizable to the national population. Additional details on the HBSC study can be found in the 2014 Canadian HBSC report (Freeman et al. 2016).

\section{Measures of risk behaviour}

As per existing precedents, we defined risk behaviours as "voluntary behaviours having known health consequences that can threaten an individual's successful physical and/or psychosocial development", acknowledging that risk behaviours can also be part of "normal adolescent development" (Jessor 1991). All risk behaviours that met this definition and were measured in 
Cycle 7 of the Canadian HBSC were identified. We then categorized each identified risk behaviour (28 identified in total) according to the six domains of risk as outlined in the CDC framework (Kann et al. 2016). To standardize our approach to classification and subsequent factor analysis for categorical variables, we re-coded each of the 28 items into three broad categories based on level of behavioural engagement and group size: low (no or minimal engagement in the risk behaviour), medium, and high (extensive engagement) (see Table 1). A combination of current and lifetime exposure to risk behaviours was studied. This was done intentionally to capture a student's propensity to engage in certain behaviours earlier in life that might lead to subsequent engagement into different behaviours.

\section{Statistical analyses}

Latent risk constructs were identified from the list of risk behaviours and then validated using exploratory and confirmatory factor analyses, respectively (Kline 2013). A split-sampling method was followed, with the study sample randomly divided in half using a simple random sampling technique (equal probability without replacement). Separate exploratory and confirmatory factor analyses were then performed for each of the two grade groups $(6-8,9-10)$ due to the differences in the available measures of risk behaviours in the two groups. Common factors in the exploratory analyses were extracted using iterated principal axis factoring with promax rotation. Factor loadings below 0.30 were suppressed (Kline 1994). Factor interpretability, scree plots, and parallel analyses (Kabacoff 2003) were used to specify the number of factors to include in the final model (Fabrigar et al. 1999). Confirmatory factor analysis using maximum-likelihood estimation was used with the second group in an attempt to validate the common factor structure. Root-mean-squared error of approximation (RMSEA), standardized root-mean-square residuals (SRMR), and adjusted goodness-of-fit index (AGFI) were used to evaluate model fit (Hooper et al. 2008). Intraclass correlations were calculated separately for all risk behaviours included in the final models to assess for clustering at the school level. Direct correlations and correlations corrected for attenuation (Schmitt 1996) were calculated between identified subscales, and McDonald's omega was calculated to assess the internal consistency (reliability) for each subscale (Zhang and Yuan 2015). All analyses in this study considered sample weights and were conducted in SAS (Version 9.4, SAS Institute, Cary, NC). McDonald's omega values were calculated using $\mathrm{R}$ (Version 3.4.1, R Foundation for Statistical Computing, Vienna, Austria).

\section{Results}

\section{Sample population}

Of the 30,096 responses available for study, 13,806 were in Grades $9-10$ and 16,290 were in Grades 6-8. The proportion of students identified as being in the high-risk category for each of the risk behaviours included in the final model can be found in Table 2 .

\section{Exploratory and confirmatory factor analysis}

After consideration of the available 28 risk behaviours, a three-factor solution emerged from the exploratory factor analyses within both grade groups. The final model in both grade groups had an independent cluster solution. Eigenvalues for all common factors in the models were above the $90 \%$ confidence intervals from the parallel analyses, suggesting that variances explained by the factors were better than a chance finding. Final eigenvalues for the Grades 9-10 model were 5.08, 2.01, and $1.13(N=3594)$. For Grades $6-8$ students, findings were consistent; the final eigenvalues were $3.06,1.65$, and $1.08(N=5586)$. Only students with responses to all risk behaviours were included in the factor analyses. Given the reduced sample sizes used for the final exploratory factor analyses due to missing responses, sensitivity analyses using full information maximumlikelihood imputation were performed, and no significant changes to factor structure, eigenvalues, or factor loadings were identified.

Based on similar results from the exploratory analyses for both grade groups, the three risk behaviour categories were labeled together based on a general conceptual understanding of the behaviours that emerged from their respective exploratory factor analyses (Tables 3,4$)$. The first common factor showed behaviours associated with substance use and externalizing risk-taking such as fighting, non-helmet use while riding on a bicycle, and risky sexual behaviour. We called this category 'Overt Risk Taking'. The second factor identified behaviours associated with low consumption of nutritious food (such as fruits and vegetables) and low participation in different forms of moderateto-vigorous physical activity (such as organized sports and free play). Because of the omission of behaviours associated with healthy, balanced lifestyles, we called this category 'Aversion to a Healthy Lifestyle'. The third factor grouped sedentary screen time activities together with unhealthy snacking behaviours (i.e., potato chip and soda consumption)—we called this category the 'Screen Time Syndrome'. Cronbach's alpha values calculated for each of the risk behaviour categories in both grade groups were all above 0.60 suggesting acceptable levels of internal consistency. 
Table 1 Initial set of risk behaviours from the HBSC used for exploratory factor analysis (Canada 2014)

\begin{tabular}{|c|c|c|c|}
\hline Initial set of risk behaviours & $\begin{array}{l}\text { None/minimal } \\
\text { engagement }\end{array}$ & Moderate engagement & $\begin{array}{l}\text { Frequent } \\
\text { engagement }\end{array}$ \\
\hline \multicolumn{4}{|l|}{ CDC domain 1: smoking cigarettes } \\
\hline Number of days they smoked cigarettes in their life & Never & $1-29$ days & $30+$ days \\
\hline Alternative tobacco products (e.g., e-cigarette, flavored tobacco...) ${ }^{\mathrm{b}}$ & Never used any & Used one once or more & $\begin{array}{l}\text { Used several once or } \\
\text { more }\end{array}$ \\
\hline \multicolumn{4}{|l|}{ CDC domain 2: alcohol and illicit substance use } \\
\hline Frequency of alcohol consumption (e.g., beer, wine, cider... $)^{\mathrm{b}}$ & Never drank any & Rarely & $\begin{array}{l}\text { Every month-every } \\
\text { day }\end{array}$ \\
\hline Number of drinks per typical event & Never drank & Less than 1 -one drink & $2+$ drinks \\
\hline Number of times they got drunk in their life ${ }^{a}$ & Never & Once & $2+$ times \\
\hline Frequency of binge drinking in the last year ${ }^{\mathrm{a}}$ & Never drank-never binged & $\begin{array}{l}\text { Less than or once a } \\
\text { month }\end{array}$ & $\begin{array}{l}2-3 \text { times a month- } \\
\text { daily }\end{array}$ \\
\hline Number of days they used cannabis in their life $\mathrm{a}^{\mathrm{a}}$ & Never & $1-5$ days & $6+$ days \\
\hline $\begin{array}{l}\text { Number of times they used hard drugs (e.g., ecstasy, solvents, pain } \\
\text { medication... })^{\text {ab }}\end{array}$ & Never used any & Used one once & $\begin{array}{l}\text { Used several once or } \\
\text { more }\end{array}$ \\
\hline \multicolumn{4}{|l|}{ CDC domain 3: high-risk sexual behaviours } \\
\hline Lifetime sexual history and use of contraceptives ${ }^{\mathrm{ab}}$ & Never had sex & $\begin{array}{l}\text { Had sex using } \\
\text { contraception }\end{array}$ & $\begin{array}{l}\text { Sex without } \\
\text { contraception }\end{array}$ \\
\hline \multicolumn{4}{|l|}{ CDC domain 4: high-risk manifest behaviours } \\
\hline Number of times they got into a fight in the last year & No fights & Once & $2+$ times \\
\hline Frequency of personal bullying behaviours on others & No bullying & Once-3 times a month & Once a week or more \\
\hline Frequency of helmet use while riding a bicycle & Always ${ }^{c}$ & $\begin{array}{l}\text { Sometimes-most of the } \\
\text { time }\end{array}$ & Never \\
\hline Frequency of helmet use while in an off-road vehicle & Always $^{c}$ & $\begin{array}{l}\text { Sometimes-most of the } \\
\text { time }\end{array}$ & Never \\
\hline \multicolumn{4}{|l|}{ CDC domain 5: unhealthy dietary pattern } \\
\hline Frequency of sugar-sweetened soda consumption in a typical week & Never-once a week & $2-4$ times a week & $\begin{array}{l}5-6 \text { times a week or } \\
\text { more }\end{array}$ \\
\hline Frequency of chip consumption in a typical week & Never-once a week & $2-4$ times a week & $\begin{array}{l}5-6 \text { times a week or } \\
\text { more }\end{array}$ \\
\hline Frequency of sweet/candy consumption in a typical week & Never-once a week & $2-4$ times a week & $\begin{array}{l}5-6 \text { times a week or } \\
\text { more }\end{array}$ \\
\hline Frequency of caffeinated energy drink consumption in a typical week & Never & Less than or once a week & $\begin{array}{l}2-4 \text { times a week or } \\
\text { more }\end{array}$ \\
\hline Frequency of fruit consumption in a typical week & Once or more a day & 2-6 times a week & Never-once a week \\
\hline Frequency of vegetable consumption in a typical week & Once or more a day & $2-6$ times a week & Never-once a week \\
\hline Frequency of orange vegetable consumption in a typical week & Once or more a day & 2-6 times a week & Never-once a week \\
\hline \multicolumn{4}{|l|}{ CDC domain 6: physical inactivity } \\
\hline Hours watching TV or videos on a typical day ${ }^{\mathrm{b}}$ & None- $1.5 \mathrm{~h}$ & $1.5-3 \mathrm{~h}$ & $3+$ hours \\
\hline Hours playing video games on a typical day ${ }^{b}$ & None-1.5 h & $1.5-3 \mathrm{~h}$ & $3+$ hours \\
\hline Hours using a computer on a typical day ${ }^{b}$ & None-1.5 h & $1.5-3 \mathrm{~h}$ & $3+$ hours \\
\hline Hours playing outdoors outside of school hours ${ }^{\mathrm{b}}$ & $3+$ hours & $1-3 \mathrm{~h}$ & None-1 h \\
\hline Participation in organized sports ${ }^{\mathrm{b}}$ & $\begin{array}{l}\text { Both individual and team } \\
\text { sport }\end{array}$ & $\begin{array}{l}\text { Either individual or team } \\
\text { sport }\end{array}$ & No participation \\
\hline Active travel to school (including duration) ${ }^{\mathrm{b}}$ & Walk/bike 5 min or more & $\begin{array}{l}\text { Walk/bike less than } \\
5 \mathrm{~min}\end{array}$ & $\begin{array}{l}\text { Not walking or } \\
\text { bicycling }\end{array}$ \\
\hline Hours participating in physical education at school on a typical week & $4-7 \mathrm{~h}$ & $2-3 \mathrm{~h}$ & None-1 h \\
\hline Hours of exercise outside of school hours & 4-7 times a week & 2-3 times a week & Never-once a week \\
\hline
\end{tabular}

Coding of relative risk for each of the risk behaviours is also included. Coding within each level of risk may represent an aggregate of multiple questionnaire options

HBSC Health Behaviour in School-aged Children Study, CDC Centers for Disease Control and Prevention

${ }^{a}$ Grades 6-8 students are not asked these questions in the HBSC study

${ }^{\mathrm{b}}$ Denote risk behaviours that are a composite measure combining multiple HBSC study questionnaire items

${ }^{\mathrm{c}}$ This category also includes students who did not ride a bicycle or motor vehicle 
Table 2 Students in each risk level for all risk behaviours in the final exploratory model (Canada 2014)

\begin{tabular}{|c|c|c|c|c|c|c|c|}
\hline \multirow[t]{3}{*}{ Final set of risk behaviours } & \multicolumn{6}{|c|}{ Frequency of risk behaviour engagement } & \multirow[t]{3}{*}{ Missing } \\
\hline & \multicolumn{2}{|c|}{ None/minimal } & \multicolumn{2}{|c|}{ Moderate } & \multicolumn{2}{|c|}{ Frequent } & \\
\hline & No. & Row\% & No. & Row\% & No. & Row\% & \\
\hline \multicolumn{8}{|l|}{ Grades $9-10$ students } \\
\hline \multicolumn{8}{|l|}{ CDC domain 1: smoking cigarettes } \\
\hline Lifetime smoking history & 10,682 & $(80.7)$ & 1759 & $(13.3)$ & 798 & $(6.0)$ & 584 \\
\hline Use of alternative tobacco products & 9784 & $(74.1)$ & 1896 & $(14.4)$ & 1519 & $(11.5)$ & 624 \\
\hline \multicolumn{8}{|l|}{$\begin{array}{l}\text { CDC domain 2: alcohol and illicit } \\
\text { substance use }\end{array}$} \\
\hline Frequency of alcohol consumption & 5437 & $(40.8)$ & 4192 & $(31.5)$ & 3701 & $(27.8)$ & 492 \\
\hline Number of drinks per typical event & 6022 & $(45.8)$ & 2704 & $(20.6)$ & 4409 & $(33.6)$ & 688 \\
\hline Lifetime drunkenness history & 8676 & $(65.3)$ & 3139 & $(23.6)$ & 1465 & $(11.0)$ & 543 \\
\hline Binge drinking & 8452 & $(66.1)$ & 2968 & $(23.2)$ & 1363 & $(10.7)$ & 1040 \\
\hline Illicit drug use & 10,256 & $(77.7)$ & 594 & $(4.5)$ & 2351 & $(17.8)$ & 622 \\
\hline Lifetime cannabis use & 10,082 & $(76.5)$ & 793 & $(6.0)$ & 2303 & $(17.5)$ & 646 \\
\hline \multicolumn{8}{|l|}{ CDC domain 3: high-risk sexual behaviours } \\
\hline Sex and contraceptive use & 7503 & $(77.7)$ & 1828 & $(18.9)$ & 329 & $(3.4)$ & 4164 \\
\hline \multicolumn{8}{|l|}{ CDC domain 4: high-risk manifest behaviours } \\
\hline Physical fighting & 10,199 & $(75.7)$ & 1558 & $(11.6)$ & 1715 & $(12.7)$ & 352 \\
\hline Non-helmet use on a bicycle & 4961 & $(39.4)$ & 3025 & $(24.1)$ & 4593 & $(36.5)$ & 1244 \\
\hline \multicolumn{8}{|l|}{ CDC domain 5: unhealthy dietary pattern } \\
\hline Sweet consumption & 4601 & $(34.5)$ & 4510 & $(33.9)$ & 4211 & $(31.6)$ & 502 \\
\hline Sugar-sweetened soda consumption & 7868 & $(58.0)$ & 2943 & $(21.7)$ & 2747 & $(20.3)$ & 264 \\
\hline Chip consumption & 9508 & $(70.3)$ & 2646 & $(19.6)$ & 1374 & $(10.2)$ & 295 \\
\hline Caffeinated energy drink consumption & 9921 & $(72.5)$ & 2848 & $(20.8)$ & 911 & $(6.7)$ & 143 \\
\hline Low fruit consumption & 6234 & $(45.5)$ & 5928 & $(43.3)$ & 1542 & $(11.3)$ & 119 \\
\hline Low vegetable consumption & 6042 & $(44.4)$ & 5867 & $(43.1)$ & 1695 & $(12.5)$ & 219 \\
\hline Low orange vegetable consumption & 2096 & $(15.4)$ & 6174 & $(45.3)$ & 5351 & $(39.3)$ & 203 \\
\hline \multicolumn{8}{|l|}{ CDC domain 6: physical inactivity } \\
\hline Watching TV or videos & 3619 & $(28.6)$ & 3885 & $(30.7)$ & 5151 & $(40.7)$ & 1168 \\
\hline Playing video games & 5282 & $(41.8)$ & 2599 & $(20.6)$ & 4750 & $(37.6)$ & 1193 \\
\hline Using the computer & 3847 & $(30.7)$ & 2603 & $(20.8)$ & 6073 & $(48.5)$ & 1301 \\
\hline Outdoor play & 3184 & $(25.5)$ & 5086 & $(40.8)$ & 4199 & $(33.7)$ & 1355 \\
\hline Organized sport & 4127 & $(31.3)$ & 5341 & $(40.5)$ & 3730 & $(28.3)$ & 626 \\
\hline Outdoor exercise & 5605 & $(42.2)$ & 3747 & $(28.2)$ & 3926 & $(29.6)$ & 547 \\
\hline \multicolumn{8}{|l|}{ Grades $6-8$ students } \\
\hline \multicolumn{8}{|l|}{ CDC domain 1: smoking cigarettes } \\
\hline Lifetime smoking history & 14,730 & $(94.6)$ & 626 & $(4.0)$ & 216 & $(1.4)$ & 723 \\
\hline Use of alternative tobacco products & 14,342 & $(92.5)$ & 745 & $(4.8)$ & 417 & $(2.7)$ & 790 \\
\hline \multicolumn{8}{|l|}{$\begin{array}{l}\text { CDC domain 2: alcohol and illicit } \\
\text { substance use }\end{array}$} \\
\hline Frequency of alcohol consumption & 11,050 & $(70.7)$ & 3278 & $(21.0)$ & 1293 & $(8.3)$ & 673 \\
\hline Lifetime drunkenness history & 14,290 & $(91.9)$ & 1046 & $(6.7)$ & 212 & $(1.4)$ & 746 \\
\hline \multicolumn{8}{|l|}{$\begin{array}{l}\text { CDC domain 4: high-risk manifest } \\
\text { behaviours }\end{array}$} \\
\hline Physical fighting & 10,717 & $(68.2)$ & 2285 & $(14.5)$ & 2715 & $(17.3)$ & 578 \\
\hline Non-helmet use on a bicycle & 7576 & $(48.6)$ & 4382 & $(29.3)$ & 3320 & $(22.2)$ & 1316 \\
\hline \multicolumn{8}{|l|}{ CDC domain 5: unhealthy dietary pattern } \\
\hline Sweet consumption & 6339 & $(40.9)$ & 4666 & $(30.1)$ & 4489 & $(29.0)$ & 801 \\
\hline Sugar-sweetened soda consumption & 10,550 & $(66.4)$ & 3040 & $(19.1)$ & 2297 & $(11.5)$ & 408 \\
\hline
\end{tabular}


Table 2 continued

\begin{tabular}{|c|c|c|c|c|c|c|c|}
\hline \multirow[t]{3}{*}{ Final set of risk behaviours } & \multicolumn{6}{|c|}{ Frequency of risk behaviour engagement } & \multirow[t]{3}{*}{ Missing } \\
\hline & \multicolumn{2}{|c|}{ None/minimal } & \multicolumn{2}{|c|}{ Moderate } & \multicolumn{2}{|c|}{ Frequent } & \\
\hline & No. & Row $\%$ & No. & Row $\%$ & No. & Row\% & \\
\hline Chip consumption & 11,569 & $(72.9)$ & 2693 & $(17.0)$ & 1612 & $(10.2)$ & 420 \\
\hline Caffeinated energy drink consumption & 13,285 & $(82.8)$ & 2153 & $(13.4)$ & 601 & $(3.7)$ & 255 \\
\hline Low fruit consumption & 8557 & $(53.2)$ & 6189 & $(38.5)$ & 1345 & $(8.4)$ & 203 \\
\hline Low vegetable consumption & 7674 & $(48.4)$ & 6277 & $(39.6)$ & 1901 & $(12.0)$ & 443 \\
\hline Low orange vegetable consumption & 2959 & $(18.5)$ & 7131 & $(44.7)$ & 5868 & $(36.8)$ & 336 \\
\hline \multicolumn{8}{|l|}{ CDC domain 6: physical inactivity } \\
\hline Watching TV or videos & 5449 & $(36.4)$ & 4463 & $(29.8)$ & 5043 & $(33.7)$ & 1338 \\
\hline Playing video games & 6769 & $(45.3)$ & 3473 & $(23.3)$ & 4696 & $(31.4)$ & 1357 \\
\hline Using the computer & 7161 & $(48.2)$ & 3035 & $(20.4)$ & 4668 & $(31.4)$ & 1430 \\
\hline Outdoor play & 4572 & $(30.8)$ & 6249 & $(42.1)$ & 4008 & $(27.0)$ & 1464 \\
\hline Organized sport & 5377 & $(34.7)$ & 6702 & $(43.2)$ & 3427 & $(22.1)$ & 788 \\
\hline Outdoor exercise & 8020 & $(50.3)$ & 4331 & $(27.1)$ & 3607 & (22.6) & 337 \\
\hline
\end{tabular}

Row percentages do not take missing values into consideration and may not add to $100 \%$ due to rounding

$C D C$ Centers for Disease Control and Prevention

In both grade groups, modest correlations were observed between common factors. Overt Risk Taking was marginally correlated to both Aversion to a Healthy Lifestyle (Grades 9-10: $r=0.06, r_{\text {corrected }}=0.08$; Grades 6-8: $\left.r=0.21, r_{\text {corrected }}=0.30\right)$ and the Screen Time Syndrome (Grades 9-10: $r=0.14, \quad r_{\text {corrected }}=0.19$; Grades 6-8: $r=0.21, r_{\text {corrected }}=0.32$ ). There were positive correlations of moderate strength between the Screen Time Syndrome and Aversion to a Healthy Lifestyle in both grade groups (Grades 9-10: $r=0.30, r_{\text {corrected }}=0.47$; Grades 6-8: $\left.r=0.31, r_{\text {corrected }}=0.47\right)$. However, overall these correlations suggest that these three factors are distinct from one another. Intraclass correlations (ICC) calculated for each risk behaviour were variable and suggested lowto-moderate variance attributable to the school level (Grades 9-10: ICC range $=0.011-0.096$, Table 3; Grades $6-8$ : ICC range $=0.023-0.149$, Table 4).

Confirmatory factor analyses suggested that the Grades 9-10 $($ SRMR $=0.071$, RMSEA $=0.088$, AGFI $=0.807$, $\left.N=3693 ; \chi^{2}=6660, d f=227, p<0.001\right)$ and the Grades $\quad 6-8$ final models $\quad($ SRMR $=0.067$, RMSEA $=0.074$, AGFI $=0.885, N=5984 ; \chi^{2}=4995$, $d f=149, p<0.001$ ) had modest fits (Hooper et al. 2008).

Confirmatory factor analyses were also performed to investigate a two-factor model that combined items from the Aversion to a Healthy Lifestyle and Screen Time Syndrome categories. This two-factor model performed poorer across all of the fit indices (Grades 9-10: SRMR $=0.079$, RMSEA $=0.094, \quad$ AGFI $=0.782 ; \quad$ Grades $\quad 6-8$ : SRMR $=0.083$, RMSEA $=0.094$, AGFI $=0.812)$ when compared to the three-factor model.

\section{Discussion}

This study provides a contemporary and comprehensive examination of risk-taking behaviours among Canadian adolescents-a population that is vital to examine in the field of multiple risk behaviour as it is a critical period of the life course. The objective of this study was to evaluate relationships between risk behaviours, both novel and contemporary, derived from a list inspired by the diverse domains of the CDC framework. We conducted this analysis within a Canadian context. Based on that objective, we found that three composite indicators of clustered risk behaviour emerged from our analysis. Interestingly, these new latent constructs encompassed behaviours that crossed all six domains of risk described within the CDC framework. Our findings were fairly consistent across two broad developmental periods (Grades 6-8 and 9-10), although the items available to measure adolescent risk-taking were limited in the youngest age group.

The CDC's Youth Risk Behaviour Survey of adolescent risk behaviour evaluates and monitors the domains of behaviour most closely associated with known leading causes of morbidity and mortality (Kann et al. 2016). Continuous revision based on public health data and expert opinion means that it consistently captures a comprehensive list of new and long-standing risk behaviours that impact youth, which makes it a frequently used tool for prevention and harm-reduction programs. However, many current public health programs remain outdated in their use of the CDC framework by targeting individual domains of behaviour (i.e., alcohol and illicit substance use), or even 
Table 3 Exploratory factor analysis on risk behaviours considering all domains in the US Centers for Disease Control and Prevention risk framework in Grades 9-10 students $(N=3594)$ (Canada 2014)

\begin{tabular}{|c|c|c|c|c|}
\hline Risk behaviours & $\begin{array}{l}\text { Factor } 1 \\
\text { Overt Risk } \\
\text { Taking }\end{array}$ & $\begin{array}{l}\text { Factor } 2 \\
\text { Aversion to a } \\
\text { Healthy Lifestyle }\end{array}$ & $\begin{array}{l}\text { Factor } 3 \\
\text { Screen Time } \\
\text { Syndrome }\end{array}$ & $\begin{array}{l}\text { Intraclass } \\
\text { correlation }\end{array}$ \\
\hline \multicolumn{5}{|l|}{ CDC domain 1: smoking cigarettes } \\
\hline Lifetime smoking history & 0.65 & & & 0.062 \\
\hline Use of alternative tobacco products & 0.71 & & & 0.048 \\
\hline \multicolumn{5}{|l|}{$\begin{array}{l}\text { CDC domain 2: alcohol and illicit } \\
\text { substance use }\end{array}$} \\
\hline Frequency of alcohol consumption & 0.76 & & & 0.080 \\
\hline Number of drinks per typical event & 0.80 & & & 0.096 \\
\hline Lifetime drunkenness history & 0.85 & & & 0.065 \\
\hline Binge drinking & 0.81 & & & 0.061 \\
\hline Illicit drug use & 0.33 & & & 0.084 \\
\hline Lifetime cannabis use & 0.73 & & & 0.050 \\
\hline \multicolumn{5}{|l|}{ CDC domain 3: high-risk sexual behaviours } \\
\hline Sex and contraceptive use & 0.46 & & & 0.045 \\
\hline \multicolumn{5}{|l|}{ CDC domain 4: high-risk manifest behaviours } \\
\hline Non-helmet use on a bicycle & 0.32 & & & 0.077 \\
\hline \multicolumn{5}{|l|}{ CDC domain 5: unhealthy dietary pattern } \\
\hline Caffeinated energy drink consumption & 0.37 & & & 0.022 \\
\hline \multicolumn{5}{|l|}{ CDC domain 5: unhealthy dietary pattern } \\
\hline Fruit consumption & & 0.63 & & 0.040 \\
\hline Vegetable consumption & & 0.56 & & 0.039 \\
\hline Orange vegetable consumption & & 0.56 & & 0.020 \\
\hline \multicolumn{5}{|l|}{ CDC domain 6: physical inactivity } \\
\hline Duration of outdoor play & & 0.39 & & 0.041 \\
\hline Participation in organized sports & & 0.48 & & 0.024 \\
\hline Frequency of exercise outside school hours & & 0.47 & & 0.026 \\
\hline \multicolumn{5}{|l|}{ CDC domain 5: unhealthy dietary pattern } \\
\hline Sweet consumption & & & 0.39 & 0.011 \\
\hline Sugar-sweetened soda consumption & & & 0.59 & 0.036 \\
\hline Chip consumption & & & 0.51 & 0.015 \\
\hline \multicolumn{5}{|l|}{ CDC domain 6: physical inactivity } \\
\hline Watching TV or videos & & & 0.38 & 0.014 \\
\hline Playing video games & & & 0.44 & 0.014 \\
\hline Using a computer & & & 0.31 & 0.018 \\
\hline Final eigenvalues & 5.08 & 2.01 & 1.13 & \\
\hline Cronbach's alpha (standardized) & 0.87 & 0.67 & 0.61 & \\
\hline McDonald's omega & 0.88 & 0.54 & 0.57 & \\
\hline $\begin{array}{l}\text { Confirmatory factor analysis }{ }^{\mathrm{a}}: \text { RMSEA } \\
(90 \% \mathrm{CI})\end{array}$ & \multicolumn{4}{|c|}{$0.088(0.086,0.089)$} \\
\hline Confirmatory factor analysis ${ }^{\mathrm{a}}$ : SRMR & 0.071 & & & \\
\hline Confirmatory factor analysis ${ }^{\mathrm{a}}$ : AGFI & 0.807 & & & \\
\hline
\end{tabular}

Exploratory factor analysis using iterated principal axis factoring and promax rotation. Factor loadings lower than 0.3 were suppressed

$C D C$ Centers for Disease Control and Prevention, RMSEA root-mean-square error of approximation, SRMR standard root-mean-square residual, $A G F I$ adjusted goodness-of-fit index

${ }^{\text {a } C o n f i r m a t o r y ~ f a c t o r ~ a n a l y s i s ~ u s i n g ~ m a x i m u m-l i k e l i h o o d ~ e s t i m a t i o n ~}$ 
Table 4 Exploratory factor analysis on risk behaviours considering all domains in the US Centers for Disease Control and Prevention risk framework in Grades 6-8 students $(N=5586)$ (Canada 2014)

\begin{tabular}{|c|c|c|c|c|}
\hline Risk behaviours & $\begin{array}{l}\text { Factor 1 } \\
\text { Overt Risk } \\
\text { Taking }\end{array}$ & $\begin{array}{l}\text { Factor } 2 \\
\text { Aversion to a } \\
\text { Healthy Lifestyle }\end{array}$ & $\begin{array}{l}\text { Factor } 3 \\
\text { Screen Time } \\
\text { Syndrome }\end{array}$ & $\begin{array}{l}\text { Intraclass } \\
\text { correlation }\end{array}$ \\
\hline \multicolumn{5}{|l|}{ CDC domain 1: smoking cigarettes } \\
\hline Lifetime smoking history & 0.73 & & & 0.047 \\
\hline Use of alternative tobacco products & 0.76 & & & 0.067 \\
\hline \multicolumn{5}{|l|}{ CDC domain 2: alcohol and illicit substance use } \\
\hline Frequency of alcohol consumption & 0.57 & & & 0.074 \\
\hline Lifetime drunkenness history & 0.65 & & & 0.050 \\
\hline \multicolumn{5}{|l|}{ CDC domain 4: high-risk manifest behaviours } \\
\hline Non-helmet use on a bicycle & 0.35 & & & 0.149 \\
\hline Physical fighting & 0.30 & & & 0.029 \\
\hline \multicolumn{5}{|l|}{ CDC domain 5: unhealthy dietary pattern } \\
\hline Caffeinated energy drink consumption & 0.42 & & & 0.046 \\
\hline \multicolumn{5}{|l|}{ CDC domain 5: unhealthy dietary pattern } \\
\hline Fruit consumption & & 0.62 & & 0.045 \\
\hline Vegetable consumption & & 0.61 & & 0.053 \\
\hline Orange vegetable consumption & & 0.51 & & 0.028 \\
\hline \multicolumn{5}{|l|}{ CDC domain 6: physical inactivity } \\
\hline Duration of outdoor play & & 0.36 & & 0.052 \\
\hline Participation in organized sports & & 0.38 & & 0.034 \\
\hline Frequency of exercise outside school hours & & 0.48 & & 0.029 \\
\hline \multicolumn{5}{|l|}{ CDC domain 5: unhealthy dietary pattern } \\
\hline Sweet consumption & & & 0.37 & 0.023 \\
\hline Sugar-sweetened soda consumption & & & 0.50 & 0.066 \\
\hline Chip consumption & & & 0.43 & 0.034 \\
\hline \multicolumn{5}{|l|}{ CDC domain 6: physical inactivity } \\
\hline Watching TV or videos & & & 0.50 & 0.029 \\
\hline Playing video games & & & 0.55 & 0.029 \\
\hline Using a computer & & & 0.50 & 0.043 \\
\hline Final eigenvalues & 3.06 & 1.65 & 1.08 & \\
\hline Cronbach's alpha (standardized) & 0.75 & 0.65 & 0.66 & \\
\hline McDonald's omega & 0.68 & 0.63 & 0.63 & \\
\hline Confirmatory factor analysis ${ }^{\mathrm{a}}$ : RMSEA $(90 \% \mathrm{CI})$ & \multicolumn{4}{|c|}{$0.074(0.072,0.076)$} \\
\hline Confirmatory factor analysis ${ }^{\mathrm{a}}: \mathrm{SRMR}$ & \multicolumn{4}{|l|}{0.067} \\
\hline Confirmatory factor analysis ${ }^{\mathrm{a}}$ : AGFI & \multicolumn{4}{|l|}{0.885} \\
\hline
\end{tabular}

Exploratory factor analysis using iterated principal axis factoring and promax rotation. Factor loadings lower than 0.3 were suppressed $C D C$ Centers for Disease Control and Prevention, RMSEA root-mean-square error of approximation, SRMR standard root-mean-square residual, $A G F I$ adjusted goodness-of-fit index

${ }^{\mathrm{a} C}$ Confirmatory factor analysis using maximum-likelihood estimation

individual risk behaviours within a domain (i.e., alcohol consumption) for public health interventions and ignore the well-established concept of their inter-related and clustered natures. By incorporating lessons of Problem Behaviour Theory (Jessor 1991, 2014), our study uses the comprehensive CDC risk domains to identify three clusters of risk behaviour that may provide more focused public health interventions targeting contemporary populations of young Canadians.
Our approach to conceptualizing risk behaviour recognizes the complex relationships that exist amongst them, as well as their possible joint effects on disease etiology. The items indicated by the CDC domains are intimately related in interpretable and potentially unexpected ways, consistent across grade groups. As a result, these behaviours should be observed and measured collectively under each of the three categories to be properly understood and managed. 
The three risk categories that were identified by factor analysis incorporated items that are well recognized within the adolescent health research literature. The Overt Risk Taking category largely encompasses behaviours found in the traditional adolescent risk studies (Maggs et al. 1997; de Looze et al. 2012). However, we believe that the benefits of our analysis lie in the more contemporary expressions of risk-taking that were incorporated. For example, the inclusion of caffeinated energy drinks and alternative tobacco products highlights emergent areas of related risk that reveal either the true breadth of this category, or behaviours associated with more moderate risk tolerance that indicate early development of risk-taking in this domain. In contrast, the traditional public health programs targeting individual behaviours, such as cigarette use, may forego the opportunity to educate and prevent other behaviours within the same category that an adolescent is likely already participating in (such as consumption of caffeinated energy drinks, alternative tobacco products, cannabis use, and riding a bicycle without a helmet). The Screen Time Syndrome and Aversion to a Healthy Lifestyle categories indicated close relationships between diet, physical activity, and sedentary behaviour that have been recognized in the past research (Leech et al. 2014). Nevertheless, our empirical distinction between these categories also supports studies that show that a lack of moderate-to-vigorous physical activity and excessive screen time represent separate and distinct behaviours among adolescent populations (Pearson et al. 2014; Brindova et al. 2015). Our study's risk categories represent measures of three separate types of adolescent risk behaviour that were found to be robust through confirmatory analysis in a separate subset of our study population.

Although psychometric research on the relationships amongst risk behaviours has been conducted in the past, none have used an established framework to ensure that they have captured a group of behaviours that are associated with the current leading causes of illness and injury among youths. Our three categories incorporate behaviours that span each of the six of the CDC risk behaviour domains, and the resultant composite scores are, perhaps, more consistent with the way that adolescents behave socially compared to other studies of multiple risk behaviours. These three risk categories have implications for the development and targeting of public health interventions that improve upon individual risk behaviour approaches, and can broaden clustered risk behaviour approaches with a narrow scope (i.e., Sloboda et al. 2009).

Of importance, our analysis extends the existing research (e.g., Pickett et al. 2002; De La Haye et al. 2014) by being amongst the first studies to factor analytically derive composite indicators of risk-taking based on an established framework. This study identified three ways that adolescents engage in risk-taking behaviours-each presumably having their own upstream determinants and downstream health consequences. Further research is now needed to confirm these as stable and consistent composite indicators of risk-taking in other study populations and contexts, and to evaluate the health outcomes and risk factors associated with each distinct category. Future intervention programs could then target the risk factors of each category to address their associated negative health outcomes (Jackson et al. 2012; Hale et al. 2014).

Admittedly, research such as this is often limited by its reliance on self-reported data. The HBSC attempts to minimize this limitation through the emphasis of confidentiality of responses (Currie et al. 2012). Nevertheless, students may not have answered truthfully to all the questions due to social desirability biases. Similarly, risk behaviour and sensitive questions have higher rates of non-response, most notably those surrounding sexual behaviour. Several of the items in the HBSC ask about days of lifetime exposure to specific risk behaviours and may misclassify newly emergent high-frequency engagement as moderate engagement. Finally, the CDC risk framework that inspired our work may not be completely applicable to the Canadian HBSC study population, based on cultural and age differences. Finally, we reported the intraclass correlation for each risk behaviour included in our composite measures and note that some behaviours showed moderate clustering effects at the school level. Although the majority of the risk behaviours in our study had negligible clustering effects at the school level, this analysis did not account for such clustering and may have overestimated variance at the student-level. Based on our reported measures, future analyses may choose to account for school-level clustering effects.

\section{Conclusion}

This study used a large sample of Canadian adolescents to evaluate relationships amongst adolescent risk behaviours. This psychometric research was inspired by the six-domain framework outlined by the CDC (Kann et al. 2016). Our empirical analysis, which included both exploratory and confirmatory factor analytic techniques, found that adolescent risk behaviours cluster in predictable patterns crossing the different CDC risk domains. Three categories of risk behaviours emerged based on the six-domain framework: (1) Overt Risk Taking, (2) Aversion to a Healthy Lifestyle, and (3) Screen Time Syndrome. These categories build on the existing studies of multiple risk behaviour, and inform research and intervention efforts aimed at preventing adolescent illness and injury. Future research could use this new framework of adolescent risk behaviour to study their upstream determinants, as well as their joint causes of negative health outcomes. 


\section{Compliance with ethical standards}

Funding and support The Public Health Agency of Canada and Health Canada funded Cycle 7 of the Health Behaviour in Schoolaged Children study in Canada. Additional support for this analysis included operating grants from the Canadian Institutes of Health Research and the Heart and Stroke Foundation of Canada (MOP 97962; PCR 101415). Jonathan Kwong was supported by a Canadian Institutes of Health Research Frederick Banting and Charles Best Canada Gradate Scholarship Masters Award, and an Empire Life Fellowship in Child Health.

Conflict of interest The authors declare no conflicts of interest.

Additional contributions The authors would like to thank the students, teachers, and school administrators involved with the HBSC for their participation in this study. The Canadian principal investigators of the HBSC study are Dr. John Freeman and Dr. William Pickett at Queen's University, Canada, and its national coordinator is Matthew King at Queen's University, Canada. The international coordinator of the HBSC survey is Dr. Candace Currie at University of St Andrews, Scotland.

Ethical approval All procedures performed in studies involving human participants were in accordance with the ethical standards of the institutional and/or national research committee and with the 1964 Helsinki declaration and its later amendments or comparable ethical standards.

Institutional approval This study was approved by the Queen's University Health Sciences and Affiliated Teaching Hospitals Research Ethics Board (IRB\#: 00001173).

Open Access This article is distributed under the terms of the Creative Commons Attribution 4.0 International License (http://crea tivecommons.org/licenses/by/4.0/), which permits unrestricted use, distribution, and reproduction in any medium, provided you give appropriate credit to the original author(s) and the source, provide a link to the Creative Commons license, and indicate if changes were made.

\section{References}

Basen-Engquist K, Edmundson EW, Parcel GS (1996) Structure of health risk behavior among high school students. J Consult Clin Psychol 64:764-775

Brener ND, Kann L, Kinchen SA et al (2004) Methodology of the youth risk behavior surveillance system. Morb Mortal Wkly Rep 53:1-13

Brindova D, Veselska ZD, Klein D et al (2015) Is the association between screen-based behaviour and health complaints among adolescents moderated by physical activity? Int J Public Health 60:139-145. doi:10.1007/s00038-014-0627-x

Currie C, Zanotti C, Morgan A, et al (2012) Social determinants of health and well-being among young people. Health Behaviour in School-aged Children (HBSC) study: international report from the 2009/2010 survey. World Health Organization. http://www. euro.who.int/en/publications/abstracts/social-determinants-ofhealth-and-well-being-among-young-people.-health-behaviourin-school-aged-children-hbsc-study. Accessed 9 Feb 2017

De La Haye K, D'Amico EJ, Miles JNV et al (2014) Covariance among multiple health risk behaviors in adolescents. PLoS ONE. doi:10.1371/journal.pone.0098141 de Looze M, van den Eijnden R, Verdurmen J et al (2012) Parenting practices and adolescent risk behavior: rules on smoking and drinking also predict cannabis use and early sexual debut. Prev Sci 13:594-604. doi:10.1007/s11121-012-0286-1

Fabrigar LR, Fabrigar LR, Wegener DT et al (1999) Evaluating the use of exploratory factor analysis in psychological research. Psychol Methods 4:272-299. doi:10.1037/1082-989X.4.3.272

Freeman J, King M, Pickett W (2016) Health behaviour in schoolaged children (HBSC) in Canada: focus on relationships. Public Health Agency of Canada. http://healthycanadians.gc.ca/publica tions/science-research-sciences-recherches/health-behaviour-chil dren-canada-2015-comportements-sante-jeunes/index-eng.php. Accessed 18 Feb 2017

Goniewicz ML, Leigh NJ, Gawron M et al (2016) Dual use of electronic and tobacco cigarettes among adolescents: a crosssectional study in Poland. Int J Public Health 61:189-197. doi:10.1007/s00038-015-0756-x

Hale DR, Fitzgerald-Yau N, Viner RM (2014) A systematic review of effective interventions for reducing multiple health risk behaviors in adolescence. Am J Public Health 104:e19-e41. doi:10. 2105/AJPH.2014.301874

Hooper D, Coughlan J, Mullen M (2008) Structural equation modeling: guidelines for determining model fit. Electron J Bus Res Methods 6:53-60

Jackson C, Geddes R, Haw S, Frank J (2012) Interventions to prevent substance use and risky sexual behaviour in young people: a systematic review. Addiction 107:733-747. doi:10.1111/j.13600443.2011.03751.x

Jessor R (1991) Risk behavior in adolescence: a psychosocial framework for understanding and action. $\mathrm{J}$ Adolesc Heal 12:597-605. doi:10.1016/1054-139X(91)90007-K

Jessor R (2014) Problem Behavior Theory: a half-century of research on adolescent behavior and development. In: Lerner RM, Petersen AC, Silbereisen RK, Brooks-Gunn J (eds) The developmental science of adolescence: history through autobiography, 1st edn. Psychology Press, New York, pp 239-256

Kabacoff RI (2003) Determining the dimensionality of data: a SAS macro for parallel analysis coders' corner. In: Proceedings of the twenty-eighth annual SAS users group international conference. pp 1-3

Kann L, McManus T, Harris WA et al (2016) Youth risk behavior surveillance-United States, 2015. Morb Mortal Wkly Rep 65:1-51

Kline P (1994) An easy guide to factor analysis. Routledge, New York

Kline RB (2013) Exploratory and CONfiRMATORY FACTOR ANALYSIS. In: Petscher Y, Schatschneider C, Compton DL (eds) Applied quantitative analysis in education and the social sciences, 1st edn. Routledge, New York, pp 169-207

Leech RM, McNaughton SA, Timperio A (2014) The clustering of diet, physical activity and sedentary behavior in children and adolescents: a review. Int J Behav Nutr Phys Act 11:1-9. doi:10. 1186/1479-5868-11-4

Lindberg LD, Boggess S, Williams S (1995) Multiple threats: the cooccurrence of teen health risk behaviors. Washington, DC

Lytle L (2002) Nutritional issues for adolescence. J Am Diet Assoc 102:S8-S12

Maggs JL, Frome PM, Eccles JS, Barber BL (1997) Psychosocial resources, adolescent risk behaviour and young adult adjustment: is risk taking more dangerous for some than others? J Adolesc 20:103-119. doi:10.1006/jado.1996.0067

Pearson N, Braithwaite RE, Biddle SJH et al (2014) Associations between sedentary behaviour and physical activity in children and adolescents: a meta-analysis. Obes Rev 15:666-675. doi:10. 1111/obr.12188

Pérez Fuentes MDC, del Molero Jurado MM, Carrión Martínez JJ et al (2016) Sensation-seeking and impulsivity as predictors of 
reactive and proactive aggression in adolescents. Front Psychol 7:1-8. doi:10.3389/fpsyg.2016.01447

Pfortner TK, De Clercq B, Lenzi M et al (2015) Does the association between different dimension of social capital and adolescent smoking vary by socioeconomic status? a pooled cross-national analysis. Int J Public Health 60:901-910. doi:10.1007/s00038015-0734-3

Pickett W, Schmid H, Boyce WF et al (2002) Multiple risk behavior and injury: an international analysis of young people. Arch Pediatr Adolesc Med 156:786-793. doi:10.1001/archpedi.156.8. 786

Riesch SK, Kedrowski K, Brown RL et al (2013) Health-risk behaviors among a sample of US pre-adolescents: types, frequency, and predictive factors. Int J Nurs Stud 50:10671079. doi:10.1016/j.ijnurstu.2012.10.012

Schane RE, Ling PM, Glantz SA (2010) Health effects of light and intermittent smoking: a review. Circulation 121:1518-1522. doi:10.1161/CIRCULATIONAHA.109.904235

Schmitt N (1996) Uses and abuses of coefficient alpha. Psychol Assess 8:350-353. doi:10.1037/1040-3590.8.4.350

Seifert SM, Schaechter JL, Hershorin ER, Lipshultz SE (2011) Health effects of energy drinks on children, adolescents, and young adults. Pediatrics 127:511-528. doi:10.1542/peds.2009-3592
Sloboda Z, Stephens RC, Stephens PC et al (2009) The adolescent substance abuse prevention study: a randomized field trial of a universal substance abuse prevention program. Drug Alcohol Depend 102:1-10. doi:10.1016/j.drugalcdep.2009.01.015

Spring B, Moller AC, Coons MJ (2012) Multiple health behaviours: overview and implications. J Public Health (Bangkok) 34(Suppl 1):i3-i10. doi:10.1093/pubmed/fdr111

Thompson K, Stockwell T, Leadbeater B, Homel J (2014) Association among different measures of alcohol use across adolescence and emerging adulthood. Addiction 109:894-903. doi:10. 1111/add.12499

Turner C, McClure R, Pirozzo S (2004) Injury and risk-taking behavior: a systematic review. Accid Anal Prev 36:93-101. doi:10.1016/S0001-4575(02)00131-8

Van Pelt DN, Clemens J, Brown B, Palacios M (2015) Where Our Students Are Educated: Measuring student enrolment in Canada. Fraser Institute. https://www.fraserinstitute.org/studies/whereour-students-are-educated-measuring-student-enrolment-in-canada. Accessed 08 Mar 2017

Yarber WL, Parrillo AV (1992) Adolescents and sexually transmitted diseases. J Sch Health 62:331-338

Zhang Z, Yuan K (2015) Robust coefficient alpha and omega with missing and non-normal data. $\mathrm{R}$ package version 0.5-23.1997 